Network Working Group

Request for Comments: 4557

Category: Standards Track
L. Zhu

K. Jaganathan

Microsoft Corporation

N. Williams

Sun Microsystems

June 2006

\title{
Online Certificate Status Protocol (OCSP) Support for Public Key Cryptography for \\ Initial Authentication in Kerberos (PKINIT)
}

Status of This Memo

This document specifies an Internet standards track protocol for the Internet community, and requests discussion and suggestions for improvements. Please refer to the current edition of the "Internet Official Protocol Standards" (STD 1) for the standardization state and status of this protocol. Distribution of this memo is unlimited.

Copyright Notice

Copyright (C) The Internet Society (2006).

Abstract

This document defines a mechanism to enable in-band transmission of Online Certificate Status Protocol (OCSP) responses in the Kerberos network authentication protocol. These responses are used to verify the validity of the certificates used in Public Key Cryptography for Initial Authentication in Kerberos (PKINIT), which is the Kerberos Version 5 extension that provides for the use of public key cryptography .

Table of Contents

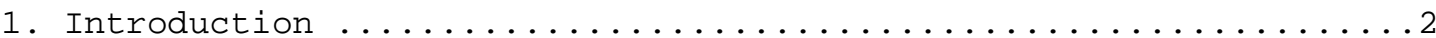

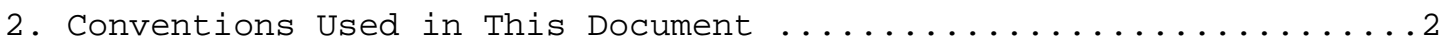

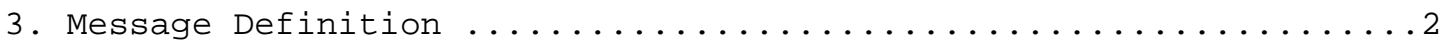

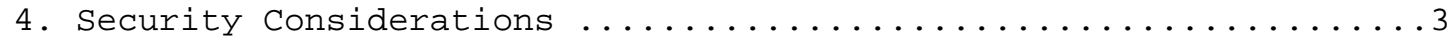

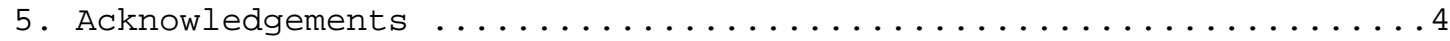

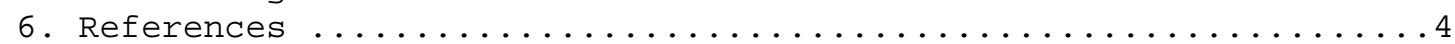

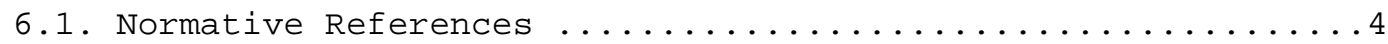

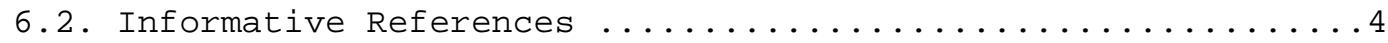




\section{Introduction}

Online Certificate Status Protocol (OCSP) [RFC2560] enables applications to obtain timely information regarding the revocation status of a certificate. Because OCSP responses are well bounded and small in size, constrained clients may wish to use ocsP to check the validity of the certificates for Kerberos Key Distribution Center (KDC) in order to avoid transmission of large Certificate Revocation Lists (CRLS) and therefore save bandwidth on constrained networks [OCSP-PROFILE] .

This document defines a pre-authentication type [RFC4120], where the client and the KDC MAY piggyback OCSP responses for certificates used in authentication exchanges, as defined in [RFC4556].

By using this OPTIONAL extension, PKINIT clients and the KDC can maximize the reuse of cached OCSP responses.

2. Conventions Used in This Document

In this document, the key words "MUST", "MUST NOT", "REQUIRED", "SHALL", "SHALL NOT", "SHOULD", "SHOULD NOT", "RECOMMENDED", "MAY", and "OPTIONAL" are to be interpreted as described in [RFC2119].

3. Message Definition

A pre-authentication type identifier is defined for this mechanism:

$$
\text { PA-PK-OCSP-RESPONSE }
$$

The corresponding padata-value field [RFC4120] contains the DER [X60] encoding of the following ASN.1 type:

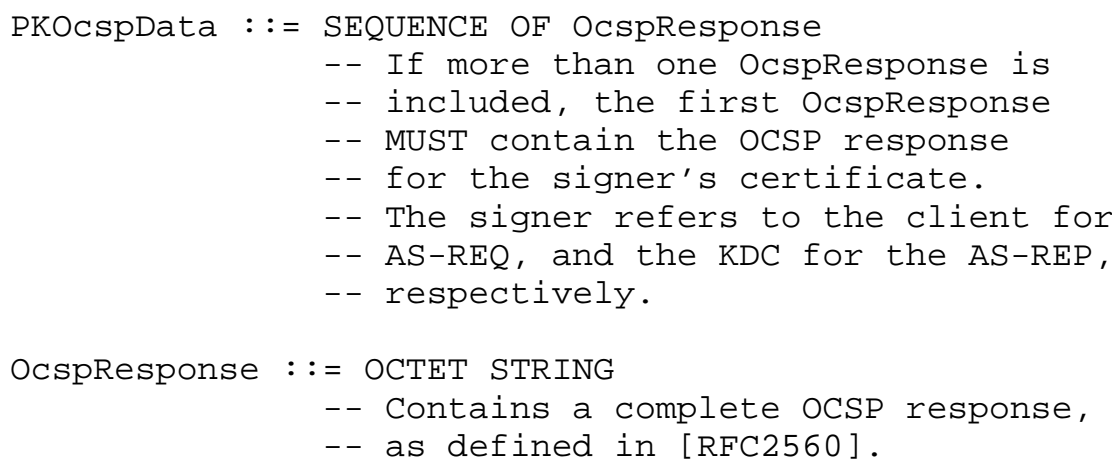

The client MAY send OCSP responses for certificates used in PA-PKAS-REQ [RFC4556] via a PA-PK-OCSP-RESPONSE. 
The KDC that receives a PA-PK-OCSP-RESPONSE SHOULD send a PA-PKOCSP-RESPONSE containing OCSP responses for certificates used in the KDC'S PA-PK-AS-REP. The client can request a PA-PK-OCSP-RESPONSE by using a PKOcspData containing an empty sequence.

The KDC MAY send a PA-PK-OCSP-RESPONSE when it does not receive a PA-PK-OCSP-RESPONSE from the client.

The PA-PK-OCSP-RESPONSE sent by the KDC contains OCSP responses for certificates used in PA-PK-AS-REP [RFC4556].

Note the lack of integrity protection for the empty or missing OCSP response; lack of an expected OCSP response from the KDC for the KDC's certificates SHOULD be treated as an error by the client, unless it is configured otherwise.

When using OCSP, the response is signed by the OCSP server, which is trusted by the receiver. Depending on local policy, further verification of the validity of the OCSP servers may be needed

The client and the KDC SHOULD ignore invalid OCSP responses received via this mechanism, and they MAY implement CRL processing logic as a fall-back position, if the OCSP responses received via this mechanism alone are not sufficient for the verification of certificate validity. The client and/or the KDC MAY ignore a valid OCSP response and perform its own revocation status verification independently.

4. Security Considerations

The pre-authentication data in this document do not actually authenticate any principals, but are designed to be used in conjunction with PKINIT.

There is no binding between PA-PK-OCSP-RESPONSE pre-authentication data and PKINIT pre-authentication data other than a given OCSP response corresponding to a certificate used in a PKINIT preauthentication data element. Attacks involving removal or replacement of PA-PK-OCSP-RESPONSE pre-authentication data elements are, at worst, downgrade attacks, where a PKINIT client or KDC would proceed without use of CRLs or OCSP for certificate validation, or denial-of-service attacks, where a PKINIT client or KDC that cannot validate the other's certificate without an accompanying OCSP response might reject the AS exchange or might have to download very large CRLs in order to continue. Kerberos V does not protect against denial-of-service attacks; therefore, the denial-of-service aspect of these attacks is acceptable. 
If a PKINIT client or KDC cannot validate certificates without the aid of a valid PA-PK-OCSP-RESPONSE, then it SHOULD fail the AS exchange, possibly according to local configuration.

5. Acknowledgements

This document was based on conversations among the authors, Jeffrey Altman, Sam Hartman, Martin Rex, and other members of the Kerberos working group.

6. References

6.1. Normative References

[RFC2119] Bradner, S., "Key words for use in RFCs to Indicate Requirement Levels", BCP 14, RFC 2119, March 1997.

[RFC2560] Myers, M., Ankney, R., Malpani, A., Galperin, S., and C. Adams, "X.509 Internet Public Key Infrastructure Online Certificate Status Protocol - OCSP", RFC 2560, June 1999.

[RFC4120] Neuman, C., Yu, T., Hartman, S., and K. Raeburn, "The Kerberos Network Authentication Service (V5)", RFC 4120, July 2005 .

[RFC4556] Zhu, L. and B. Tung, "Public Key Cryptography for Initial Authentication in Kerberos (PKINIT)", RFC 4556, June 2006 .

[X690] ASN.1 encoding rules: Specification of Basic Encoding Rules (BER), Canonical Encoding Rules (CER) and Distinguished Encoding Rules (DER), ITU-T Recommendation X.690 (1997) | ISO/IEC International Standard 8825-1:1998.

\subsection{Informative References}

[OCSP-PROFILE] Deacon, A. and R. Hurst, "Lightweight OCSP Profile for High Volume Environments", Work in Progress, May 2006. 


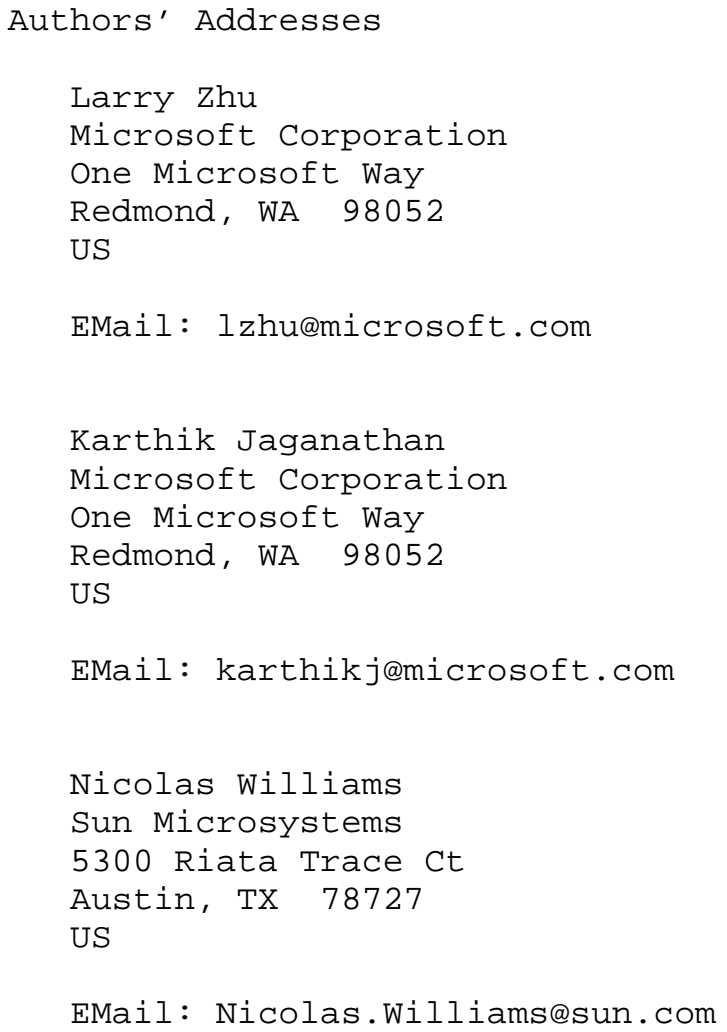


Full Copyright statement

Copyright (C) The Internet Society (2006).

This document is subject to the rights, licenses and restrictions contained in BCP 78, and except as set forth therein, the authors retain all their rights.

This document and the information contained herein are provided on an "AS IS" basis and THE CONTRIBUTOR, THE ORGANIZATION HE/SHE REPRESENTS OR IS SPONSORED BY (IF ANY), THE INTERNET SOCIETY AND THE INTERNET ENGINEERING TASK FORCE DISCLAIM ALL WARRANTIES, EXPRESS OR IMPLIED, INCLUDING BUT NOT LIMITED TO ANY WARRANTY THAT THE USE OF THE INFORMATION HEREIN WILL NOT INFRINGE ANY RIGHTS OR ANY IMPLIED WARRANTIES OF MERCHANTABILITY OR FITNESS FOR A PARTICULAR PURPOSE.

Intellectual Property

The IETF takes no position regarding the validity or scope of any Intellectual Property Rights or other rights that might be claimed to pertain to the implementation or use of the technology described in this document or the extent to which any license under such rights might or might not be available; nor does it represent that it has made any independent effort to identify any such rights. Information on the procedures with respect to rights in RFC documents can be found in BCP 78 and BCP 79 .

Copies of IPR disclosures made to the IETF Secretariat and any assurances of licenses to be made available, or the result of an attempt made to obtain a general license or permission for the use of such proprietary rights by implementers or users of this specification can be obtained from the IETF on-line IPR repository at http://www.ietf.org/ipr.

The IETF invites any interested party to bring to its attention any copyrights, patents or patent applications, or other proprietary rights that may cover technology that may be required to implement this standard. Please address the information to the IETF at ietf-ipreietf.org.

Acknowledgement

Funding for the RFC Editor function is provided by the IETF Administrative Support Activity (IASA). 